\title{
Industrial wireless sensor networks: channel modeling and performance evaluation
}

\author{
Michael Cheffena
}

\begin{abstract}
A complete dynamic wideband channel model for industrial wireless sensor network is presented. The model takes into account the noise, interferences, and heavy multipath propagation effects present in harsh industrial environments. A first-order two-state Markov process is adopted to describe the typical bursty nature of the impulsive noise usually present in industrial environments. The interference effects are modeled as multiple narrowband signals operating on the same frequency band as the desired signal. The multipath propagation is described by assuming the scatterers to be uniformly distributed in space within an elliptical region where the transmitting and receiving nodes are located at the foci of the ellipse. Furthermore, performance evaluations of IEEE 802.15.4 in terms of bit error rate using the developed channel model are presented. The results show that in addition to spread spectrum techniques, link diversity can further improve the link quality in harsh industrial environments.
\end{abstract}

Keywords: Industrial wireless sensor network, Channel model, WSN, IEEE 802.15.4

\section{Introduction}

In recent years, wireless sensor networks (WSNs) have gained worldwide attention for use in different applications. Sensor nodes are spatially distributed across a large area of interest to sense, measure, and gather information and transmit the data to the user. The nodes are typically equipped with radio transceivers, micro-controllers, and batteries. They are small in size, inexpensive, and could be deployed in large numbers. They can be used in applications such as military target tracking and surveillance $[1,2]$, natural disaster relief [3], biomedical health monitoring $[4,5]$, and industrial automation $[6,7]$.

The uses of WSN for industrial applications are expected to open large opportunities for collecting data, enabling remote control, and automation to improve the safety and productivity of facilities. Unlike wire-based systems, WSNs can be deployed in bearings of motors, oil pumps, whirring engines, or other inaccessible or hazardous environments [8]. In general, wireless solutions are considered to be cheaper compared to wire-based systems. This is due to the cost associated in shielding wires to prevent severe conditions which are usually present

Correspondence: michael.cheffena@hig.no

Gjøvik University College, Teknologivn. 22, N-2815 Gjøvik, Norway in these harsh environments (high humidity, high temperature, strong vibration, etc.) [9]. Short-range wireless technologies such as IEEE 802.15.4 [10] in mesh network configuration are widely considered to be cost effective solution for use in industrial settings.

In harsh industrial environments, noise is significant due to the wide operating temperatures, strong vibrations, excessive electromagnetic noise caused by large motors, etc. [8]. Interferences from other wireless systems operating on the same frequency band might also be present. In addition, the signal might be subject to heavy multipath propagation effects caused by multiple reflections from mainly metallic structures in the surrounding environment. The random/periodic movement of people, robots, trucks, and other objects may also cause time varying channel conditions. Effects of the aforementioned propagation impairments to mission-critical signals in industrial settings can result in costly disasters in terms of money, manpower, time, and even human lives. Thus, knowledge of the propagation channel is needed to successfully design and evaluate robust WSNs for industrial applications.

Discussions on challenges, design principles, and technical approaches of WSNs for industrial applications are presented in [11]. Radio frequency (RF) measurement results of industrial WSNs are reported in [6,12-14]. In

\section{Springer}

(c) 2012 Cheffena; licensee Springer. This is an Open Access article distributed under the terms of the Creative Commons Attribution License (http://creativecommons.org/licenses/by/2.0), which permits unrestricted use, distribution, and reproduction in any medium, provided the original work is properly cited. 
[15], the path loss and the root mean square delay spread of the signal in industrial environment were investigated. Based on measurement results, analyses of the connectivity capabilities of the IEEE 802.15.4/ZigBee technologies for mobile sensing applications in industrial environment, and their use in collision avoidance applications are reported in [16]. A framework that addresses the quality of service assessment and management of industrial WSNs is given in [17]. To the best of author's knowledge, there exist no complete dynamic wideband channel models which take into account the noise, interferences, and heavy multipath propagation effects present in harsh industrial environments. In this article, a novel complete dynamic wideband channel model which takes into account the aforementioned propagation effects usually present in harsh industrial environments is presented. In addition, performance evaluations of industrial WSN based on IEEE 802.15.4 physical layer standard are carried out using the developed channel model. In general, the proposed model can be used for generating time series which can be used for simulating and successfully designing robust WSNs for industrial applications.

The rest of the article is organized as follows. "Propagation channel characteristics of industrial WSN" section presents the proposed dynamic wideband channel model for industrial WSNs. Performance evaluations of WSN in industrial settings are presented in "Performance evaluation" section. Finally, conclusions are given in last section.

\section{Propagation channel characteristics of industrial WSN}

\section{Noise and interference}

Noise

Usually the noise affecting a given wireless communication system is modeled as additive white Gaussian noise (AWGN). However, in harsh industrial environments, wireless communication systems are also subject to impulsive noise [8-18]. The major sources of impulse noise in industrial environments are motors, heavy machineries, ignition systems, inverters, voltage regulators, electric switch contacts, welding equipments, etc. The noise in industrial environment might then be modeled as the superposition of two terms (i.e., AWGN and impulse noise), expressed as

$$
n(t)=w(t)+b(t) \cdot k(t), \quad \text { for } \quad t \in\{1,2, \ldots, T\}
$$

where $w(t)$ and $k(t)$ are zero-mean Gaussian distributed processes, $b(t)$ is a $\{0,1\}$-random variable which describes the state of the channel and $t$ is the time index. Parameter $n(t)$ describes the AWGN plus impulse noise, the former state is referred to us good state $[s(t)=G \Leftrightarrow b(t)=0]$, and the latter as bad state $[s(t)=B \Leftrightarrow b(t)=1]$. Assuming $n(t)$ is a complex circularly-symmetric
Gaussian random variable with variance depending on $s(t)$, the probability density functions of $n(t)$ conditioned to $s(t)$ are given by [19]

$$
\begin{aligned}
& P[n(t) \mid s(t)=G]=\frac{1}{2 \pi \sigma^{2}} \exp \left\{-\frac{|n(t)|^{2}}{2 \sigma^{2}}\right\} \\
& P[n(t) \mid s(t)=B]=\frac{1}{2 \pi R \sigma^{2}} \exp \left\{-\frac{|n(t)|^{2}}{2 R \sigma^{2}}\right\}
\end{aligned}
$$

where $R \geq 1$ is the ratio between the average noise power in the bad and good channels. When the channel is in a good state, the signal is impaired only by background AWGN with variance equal to $\sigma^{2}$, whereas, when in bad state, it is also impaired by impulsive noise.

The state process, $s(t)$, can be modeled as first-order two-state Markov process which describes the typical bursty nature of the impulse noise [20]

$$
P[s(t)]=P[s(1)] \prod_{t=1}^{T-1} P[s(t+1) \mid s(t)]
$$

where the evolution of the channel state is described by the transition probabilities, expressed as

$P_{i, j}=P[s(t+1)=i \mid s(t)=j], \quad$ for $\quad i, j \in\{G, B\}$

The state transitions of the first-order two-state Markov process which leads to a Markov-Gaussian channel model are shown in Figure 1. In the model, $P_{G}$ is the probability of good state $(\mathrm{G})$, whereas $P_{\mathrm{B}}$ is the probability of bad state $(B)$. The channel state changes from good to bad and from bad to good with $P_{\mathrm{GB}}$ and $P_{\mathrm{BG}}$ probabilities, respectively. The occurrence frequency of impulses and their burstiness can be controlled using parameters $P_{\mathrm{GB}}$ and $P_{\mathrm{BG}}$, respectively. High value of $P_{\mathrm{GB}}$ gives increased number of impulsive events while small values of $P_{\mathrm{BG}}$ results in increasing burstiness of each impulse. The amplitude of the impulse noise is controlled by parameter $R$ (defined in

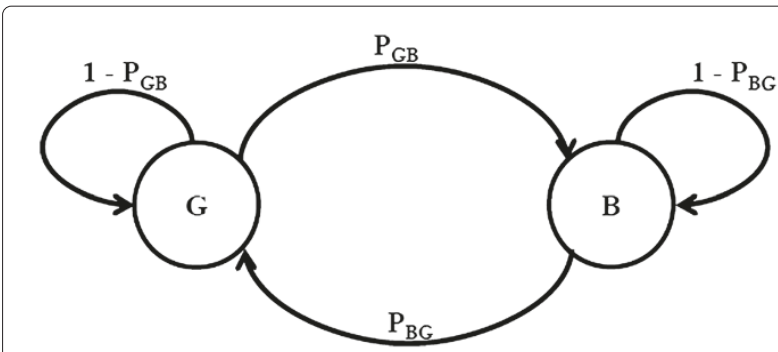

Figure 1 Two-state Markov process for modeling the typical bursty nature of the impulse noise. Parameters $G$ and $B$ are the good and bad states of the channel, respectively. Parameters $P_{\mathrm{GB}}$ and $P_{\mathrm{BG}}$ are the probability the channel changes from good to bad and from bad to good state, respectively. 
(3)) where large value of $R$ results in increased impulsive amplitude values. These model parameters need to be estimated from measurements for the considered propagation environment. Figure 2 shows examples of simulated noise time series when the channel is modeled as an AWGN (top plot) and as an AWGN plus impulse noise (bottom figure). From Figure 2, we can clearly observe that if not considered properly, impulsive noises present in harsh industrial environments could significantly degrade the performance of wireless communication systems.

\section{Interference}

Industrial WSNs can be affected by interference from other communication systems operating in the same industrial scientific medical band. The interference might be from multiple narrowband signals. The baseband expression of the interfering signals is given by

$$
J(t)=\sum_{n=0}^{N-1} \sqrt{2} \alpha_{n} \exp \left\{j \phi_{n}(t)\right\}
$$

where $\alpha_{n}$ is independent and identically distributed (i.i.d.) with $E\left[\alpha_{n}^{2}\right]=J / N$ where $J$ is the total interference power and $N$ is the total number of interfering signals. Parameter $\phi_{n}(t)$ is the phase of the $n$th interfering signal uniformly distributed within the range $[0,2 \pi)$.

The IEEE 802.15.4 uses spread spectrum techniques such as direct sequence spread spectrum (DSSS) and frequency hopping spread spectrum to reduce the effect of interference. However, for mission-critical signals in industrial settings, other strategies such as link diversity should be incorporated with the radio modulation technique to improve the link quality in these harsh environments.
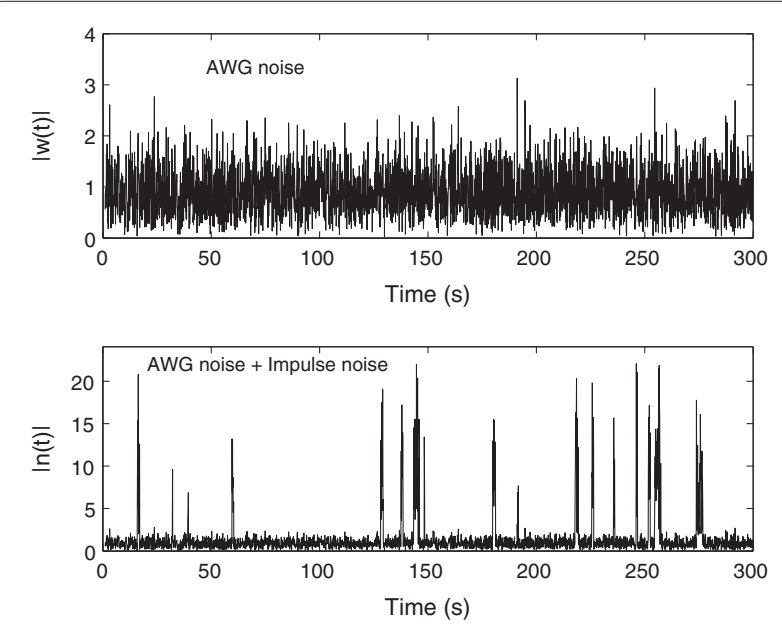

Figure 2 Simulated time series when the channel is modeled as AWGN, $w(t)$ (top plot) and as an AWGN plus impulse noise, $n(t)$ (bottom plot). The simulation parameters are $P_{\mathrm{GB}}=0.005$, $P_{\mathrm{BG}}=0.1, \sigma^{2}=1$ and $R=100$.

\section{Multipath propagation}

The multipath property of a channel depends on the type of the environment and the properties of the antennas involved. In industrial environments, reflection from mainly metallic structures such as machineries, pipes, etc., may result in heavy multipath propagation. In these environments, the objects reflecting the signal (scatterers) might be assumed to be uniformly distributed in space within an elliptical region with maximum delay of $\tau_{\max }$ such that $\tau_{0} \leq \tau_{m}<\tau_{\max }$ for all $\tau_{m}$. Parameter $\tau_{0}=d_{0} / c$ is the delay of the line-of-sight component where $d_{0}$ is the separation distance between the transmitter and the receiver, and $c=3 \times 10^{8} \mathrm{~m} / \mathrm{s}$ is the speed of light. Parameter $\tau_{m}$ is the delay of the $m$ th multipath component for $m=0,2, \ldots, M$ where $M$ is the total number of multipath components. The transmitting and receiving nodes are located at the foci of the ellipse (see Figure 3).

The strength of the reflected signals depend on the link configurations and material properties of the reflecting surfaces. The reflection coefficient can be calculated using [21]

$$
\begin{aligned}
& R_{N}=\frac{\cos \theta_{\text {ref }}-\sqrt{\eta-\sin ^{2} \theta_{\text {ref }}}}{\cos \theta_{\text {ref }}+\sqrt{\eta-\sin ^{2} \theta_{\text {ref }}}} \\
& R_{P}=\frac{\cos \theta_{\text {ref }}-\sqrt{\left(\eta-\sin ^{2} \theta_{\text {ref }}\right) / \eta^{2}}}{\cos \theta_{\text {ref }}+\sqrt{\left(\eta-\sin ^{2} \theta_{\text {ref }}\right) / \eta^{2}}}
\end{aligned}
$$

where $R_{N}$ and $R_{P}$ are the reflection coefficients when the electric field component is normal and parallel to the reflection plane, respectively. Parameter $\theta_{\text {ref }}$ is the angle between the incident ray and the normal to the reflecting surface. Parameter $\eta$ is the complex permittivity of the reflecting material. Furthermore, the envelopes of the multipath components are assumed to follow a Rice distribution.

In addition, the random/periodic movement of people, robots, trucks, and other objects in the propagation environment create time varying channel conditions. Characterization of the Doppler spectra is thus important for the determination of the time variance of the wireless

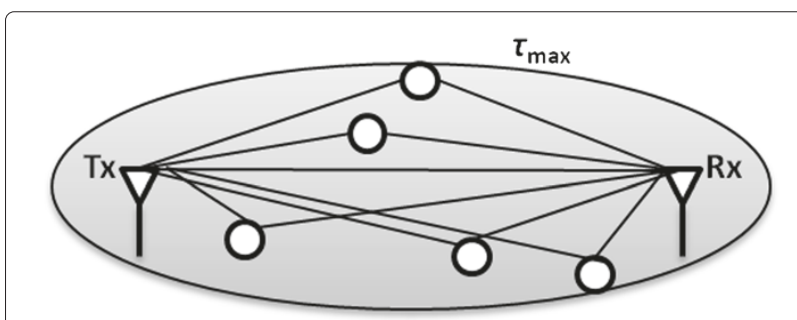

Figure 3 Uniformly distributed scatterers (represented as circles in the figure). $T x$ and $R x$ are the transmitting and receiving nodes. $\tau_{\max }$ is the maximum multipath delay. 
channel. The situation where the antenna is moving in a random environment (e.g., mobile sensor nodes) leads to the classical Jakes spectrum (with bathtub-like shape) for scatters uniformly distributed in azimuth [22].

$$
S(f)=\frac{1.5}{\pi f_{d} \sqrt{1-\left(\frac{f}{f_{d}}\right)^{2}}} \text { for }|f|<f_{d}
$$

where $f_{d}$ is the maximum Doppler shift. For the case where the antenna is stationary (i.e., static sensory nodes), moving scatterers in the channel such as people, robots, and trucks will lead to a different Doppler spectrum which peaks at $0 \mathrm{~Hz}$ and falls off rapidly [23,24]. A simplified empirical model is reported in [25], and is given by

$$
S(f)=\frac{1}{f+e}
$$

where $e$ is a model constant with value equal to 0.02 chosen to match the measured Doppler spectrum [25].

\section{Composite dynamic wideband channel model}

The time varying channel impulse response for the combined effect of noise, interferences, and multipath propagation can be expressed as

$$
\begin{aligned}
h\left(t, \tau, \theta_{\mathrm{AoD}}, \theta_{\mathrm{AoA}}\right)= & \sum_{m=0}^{M-1} A_{m}(t) \delta\left[\tau-\tau_{m}(t)\right] \\
& \delta\left[\theta_{\mathrm{AoD}}-\theta_{\mathrm{AoD}, m}(t)\right] \delta\left[\theta_{\mathrm{AoA}}-\theta_{\mathrm{AoA}, m}(t)\right] \\
& \cdot \exp \left\{j\left(\phi_{m}-\frac{2 \pi}{\lambda} d_{m}\right)\right\} \\
& +n(t)+J(t)
\end{aligned}
$$

where $\lambda$ is the wavelength. For each tap number $m, \tau_{m}(t)$ is the tap delay, $\phi_{m}$ is the phase uniformly distributed within the range $[0,2 \pi), \delta$ is delta function, $\theta_{\mathrm{AoD}}(t)$ is the angle-of-departure (AoD), $\theta_{\mathrm{AoA}}(t)$ is the angle-of-arrival (AoA), $A_{m}(t)$ is the complex amplitude, and $d_{m}$ is the path-length. Parameter $n(t)$ is the AWGN plus impulse noise as defined in (1), and $J(t)$ is the interference from multiple narrowband signals (see Equation (6)).

The path-length, delay, AoD, and AoA of each multipath component can be calculated using geometrical relation for each scatter in the elliptical region defined by the maximum tap delay, $\tau_{\max }$ (see Figure 3 ). An elliptical region offers the opportunity to limit the considered maximum delay of the multipath components [26]. Note that as defined in (7) and (8), the strength of the reflected multipath components depends on the reflection coefficients of the reflecting surfaces which in turn depend on the link configurations and material properties of the reflecting surfaces. Schematic representation of the proposed composite dynamic wideband channel model for simulating the combined propagation effects in harsh industrial environments is illustrated in Figure 4. In the model, each delayed version of the input signal, $u(t)$, is multiplied by $A_{m}(t) \exp \left\{j\left(\phi_{m}-2 \pi d_{m} / \lambda\right)\right\}$ to account for the amplitude and phase of the multipath component. The output signal, $y(t)$, is obtained by summing all multipath components and then adding the time varying noise, $n(t)$, and interference, $J(t)$ effects. Depending on the environment, the components of the model can be added or omitted to describe a given propagation condition. For example, all the model components are used in cases where the signal is affected by noise, interferences, and multipath propagation. While, in conditions where there is no RF interference, parameter $J(t)$ could be set to zero, etc.

Simulated time series of the signal-to-noise-plusinterference ratio (SNIR) for the combined effect of noise, interferences, and multipath propagation is shown in Figure 5. Isotropic antennas are used at the transmitter and the receiver. The simulation parameters are given in

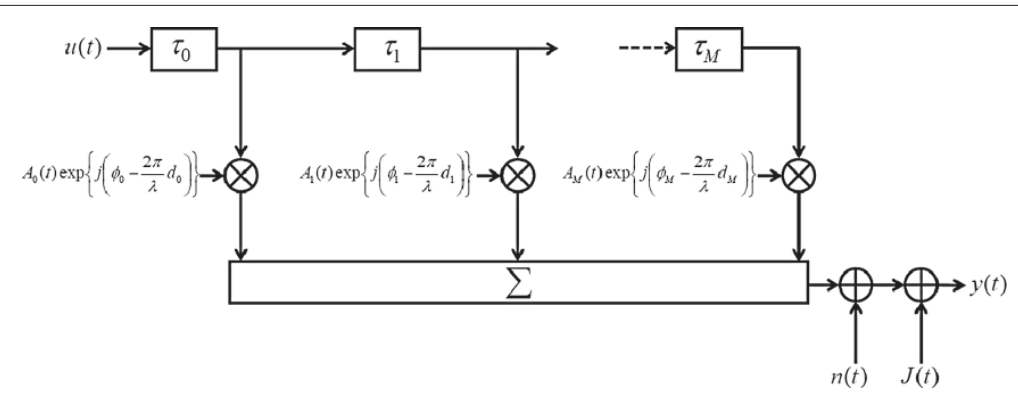

Figure 4 Dynamic wideband channel simulator for WSNs in harsh industrial environments. Parameters $u(t)$ and $y(t)$ are the input and output signals, respectively. Parameters $A(t), \tau$, and $\phi$ are the amplitude, delay, and phase of each multipath component, respectively. Parameter $\lambda$ is the wavelength, and $d$ is the path-length of each multipath component. Parameter $n(t)$ is the AWGN plus impulse noise, and $J(t)$ is the interference from multiple narrowband signals. 


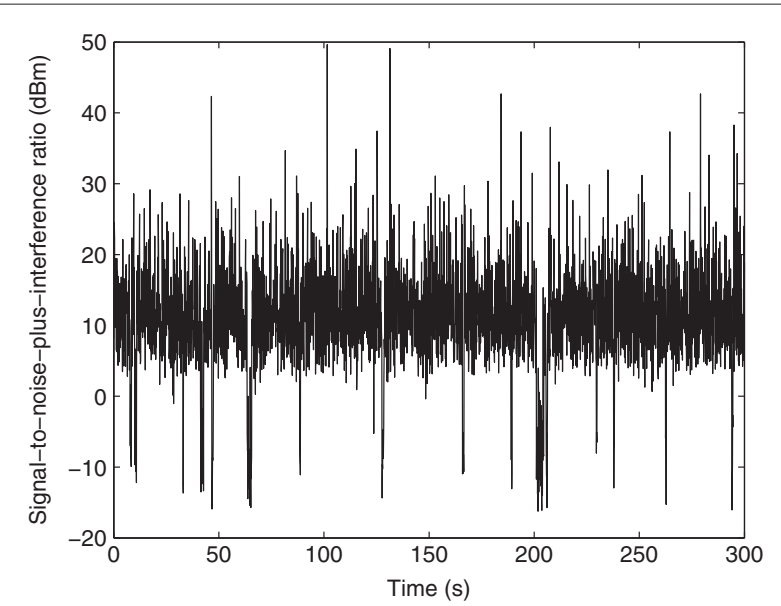

Figure 5 Simulated time series of the received SNIR for the combined effect of noise, interferences, and multipath propagation. The simulation parameters are given in Table 1.

Table 1. The multipath components are combined using a maximum-ratio-combiner (MRC) at the output of the receiver. The bursty fading events in the time series (see Figure 5) are impulsive noise effects usually present in harsh industrial environments. In addition, Figures 6, 7, and 8 show the simulated cumulative distribution functions (CDFs), level crossing rates (LCRs), and average fade durations (AFDs) of the received SNIRs at $2.4 \mathrm{GHz}$ for the composite effects of noise, interferences, and multipath propagation. The solid curves in the figures are for the cases where the noise is modeled as an AWGN $(w(t))$ while the dashed curves are for the cases when the noise

\section{Table 1 Simulation parameters}

\begin{tabular}{ll}
\hline Frequency, $f$ & $2.4 \mathrm{GHz}$ \\
Sampling frequency, $f_{\mathrm{s}}$ & $10 \mathrm{~Hz}$ \\
Simulation time, $t$ & $300 \mathrm{~s}$ \\
Transmit power, $p_{\mathrm{t}}$ & $0 \mathrm{dBm}$ \\
Distance between the Tx and the Rx, $d_{0}$ & $80 \mathrm{~m}$ \\
Receiver noise figure, $F$ & $11 \mathrm{~dB}$ \\
Receiver noise bandwidth, $B$ & $5 \mathrm{MHz}$ \\
Noise temperature, $T_{\mathrm{n}}$ & $290 \mathrm{~K}$ \\
Channel state probability from good to bad, $P_{\mathrm{GB}}$ & 0.005 \\
Channel state probability from bad to good, $P_{\mathrm{BG}}$ & 0.1 \\
Ratio of noise power in the bad and good channel, $R$ & 100 \\
Number of interfering signals, $N$ & 5 \\
Total interference power, $J$ & $4 \mathrm{dBm}$ \\
Maximum multipath delay, $\tau_{\mathrm{m}}$ & $334 \mathrm{~ns}$ \\
Number of multipath components, $M$ & 25 \\
Ricean K-factor of the multipath components, $K$ & $25 \mathrm{~dB}$ \\
Relative permittivity of the reflecting surfaces at $2.4 \mathrm{GHz}, \eta$ & $1-\mathrm{j} 802$ [21] \\
\hline
\end{tabular}

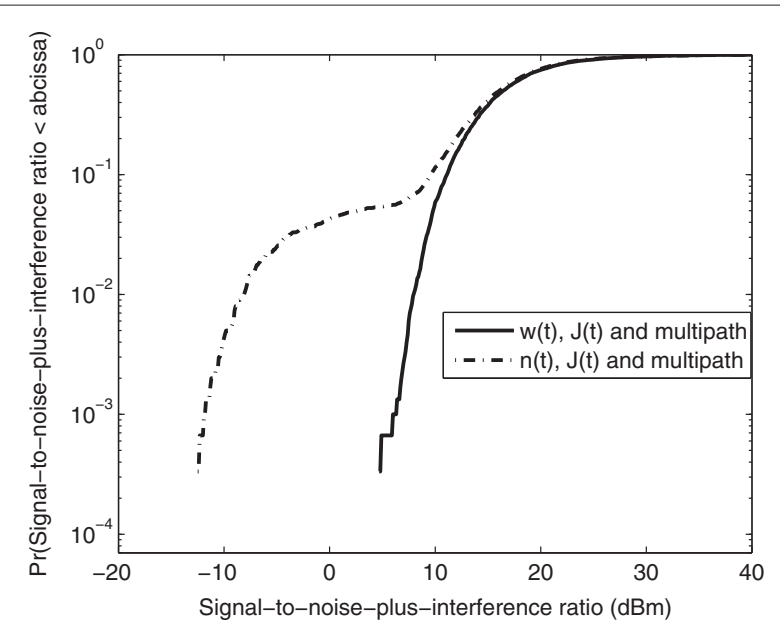

Figure 6 Simulated CDFs of the received SNIR for the combined effect of noise, interferences, and multipath propagation. Parameters $w(t), n(t)$, and $J(t)$ are as defined in (1) and (6). The simulation parameters are given in Table 1.

is modeled as an AWGN plus impulse noise $(n(t))$. From Figures 6,7 , and 8 , we can clearly observe the effect of impulsive noise on the first- and second-order statistics of the channel. These results illustrate the importance of considering the impulsive behavior of the channel when modeling the noise in harsh industrial environments.

In general, the proposed channel model can be used for generating time series which can be used for successfully designing robust industrial WSNs, and for simulating the performance of WSNs in harsh industrial environments. This enables simulation of for example

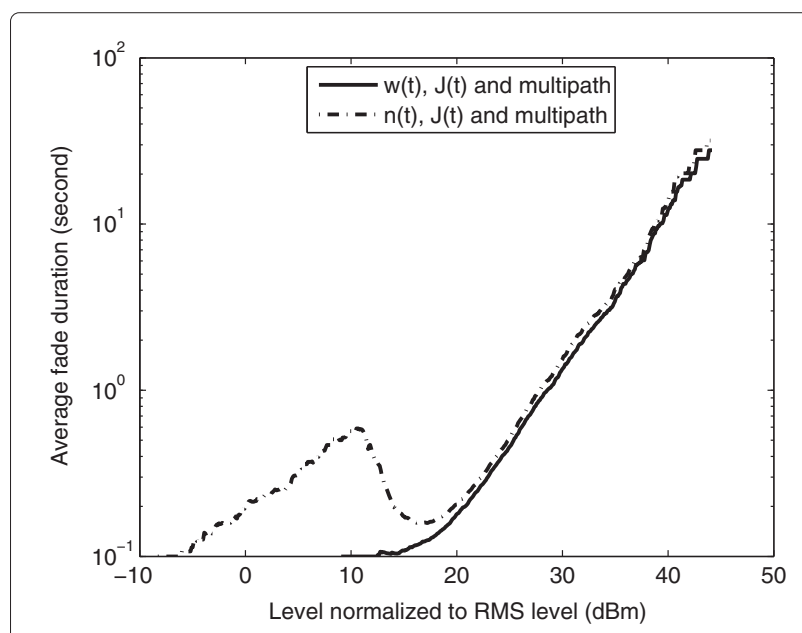

Figure 7 Simulated AFDs of the received SNIR for the combined effect of noise, interferences, and multipath propagation. Parameters $w(t), n(t)$, and $J(t)$ are as defined in (1) and (6). The simulation parameters are given in Table 1. 


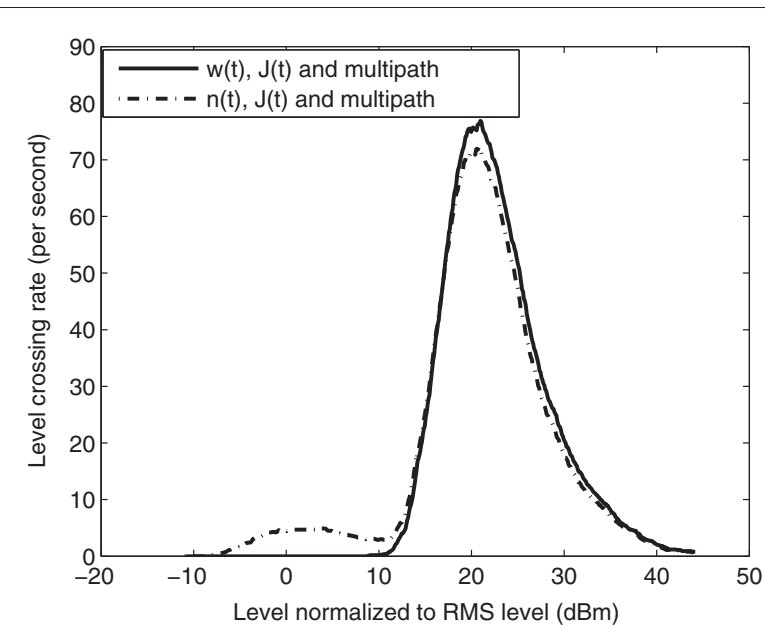

Figure 8 Simulated LCRs of the received SNIR for the combined effect of noise, interferences, and multipath propagation. Parameters $w(t), n(t)$, and $J(t)$ are as defined in (1) and (6). The simulation parameters are given in Table 1.

capacity-enhancing techniques such as route diversity and adaptive coding and modulation.

\section{Performance evaluation}

In this section, performance evaluations of IEEE 802.15.4 physical layer in harsh industrial environments using the developed dynamic wideband channel model are presented. In addition to using spread spectrum techniques for reducing the effect of interference, other strategies such as link diversity are investigated to improve the link quality in these harsh environments.

\section{Physical layer overview of IEEE 802.15.4 standard}

Short-range wireless technologies such as IEEE 802.15.4 in mesh network configuration are widely considered to be cost effective solution for use in industrial settings. The nodes consume extremely low energy and support several different topologies which makes them a good candidate for several sensor network applications.
The physical layer specification of the IEEE 802.15.4 standard is reported in [10]. It defines the characteristics of the physical layer such as spreading, despreading, modulation, and demodulation of the signal. It uses the DSSS as a spreading technique to reduce the effect of noise and interference from other networks. The IEEE 802.15.4 operates in three different frequency bands: $868 \mathrm{MHz}$ in Europe, $915 \mathrm{MHz}$ in the USA, and $2.4 \mathrm{GHz}$ globally. In the 868- and 915-MHz bands, there are three optional modulation schemes: binary phase shift keying (BPSK), offset quadrature phase shift keying (O-QPSK), and parallel sequence spread spectrum. Depending on the modulation scheme, a data rate of $250 \mathrm{kbps}$ at $2.4 \mathrm{GHz}$, from 20 to $250 \mathrm{kbps}$ at $868 \mathrm{MHz}$, and from 40 to $250 \mathrm{kbps}$ at $915 \mathrm{MHz}$ can be supported.

In the 2.4-GHz band, O-QPSK modulation and DSSS technique are used. Each 4-bit symbol is mapped to a 32chip pseudo-random noise (PN) sequence. In the 915 and $868-\mathrm{MHz}$ bands, each one-bit symbol is mapped into a 15- or 16-chip PN sequence and uses BPSK or O-QPSK for modulation, respectively. The IEEE 802.15.4 standard specifies a receiver sensitivity of $-92 \mathrm{dBm}$ in the $868 / 915$ $\mathrm{MHz}$ band and $-85 \mathrm{dBm}$ in the $2.4-\mathrm{GHz}$ band. More information can be found in [10].

\section{Bit error rate}

The composite dynamic wideband channel model presented in "Propagation channel characteristics of industrial WSN" section is used to simulate the bit error rate (BER) of WSNs in harsh industrial environments. The approach used for calculating the BER of IEEE 802.15.4 physical layer is shown in Figure 9. For the 2.4-GHz signal, integers in the range $0-15$ are drawn using a random number generator. The integers are converted to binary representations (4-bit symbols) and then sent to the spreading block which spreads them into 32 bits according to ([10], Table 20). The bit streams are taken as an input to the O-QPSK modulator and then pass thought the wireless channel (presented in "Propagation channel characteristics of industrial WSN" section) to reach the demodulator. After demodulation, the bit streams are

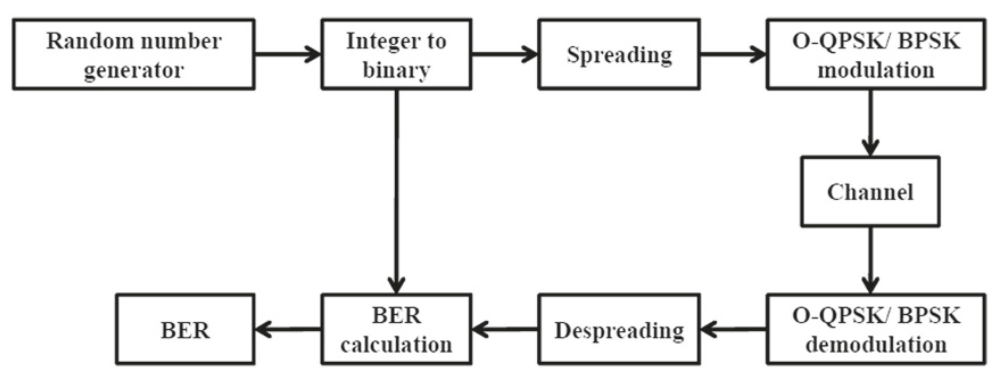

Figure 9 BER calculation for the IEEE 802.15.4 physical layer. 
passed to the despreader which converts them to 4-bit symbols. Finally, the 4-bit symbols are compared with the original one to calculate the BER.

The spread spectrum technique discussed above is used to reduce the effect of noise and interferences. However, for mission-critical signals in industrial settings, other strategies such as link diversity should be incorporated with the radio modulation technique to improve the link quality in these harsh environments. Link diversity takes advantage of the existing multipath signals in the wireless channel. In industrial environments, reflection from mainly metallic structures such as machineries, pipes, etc., may result in heavy multipath propagation which could be exploited using diversity techniques. The received SNIR can be increased by properly combining the multiple versions of the transmitted signal using, e.g., MRC. The SNIR at the output of an MRC is given by [27]

$$
\mathrm{SNIR}_{\mathrm{MRC}}=\sum_{m=0}^{M-1} \mathrm{SNIR}_{m}
$$

where $\mathrm{SNIR}_{m}$ is the SNIR of the $m$ th diversity branch and $M$ is as defined in (11).

The complementary CDFs (CCDFs) of the BERs for the combined effect of noise, interferences, and multipath propagation for single and diversity links are shown in Figure 10. The simulation parameters given in Table 1 are used. We can observe from Figure 10 that significant increase in performance can be achieved using link diversity. For example, BER of $10^{-6}$ is exceeded for about 25 and $3 \%$ of the time for the single and diversity links, respectively. Furthermore, the BER for the composite effects of noise, interferences, and multipath propagation at the output of an MRC for different burstiness of the

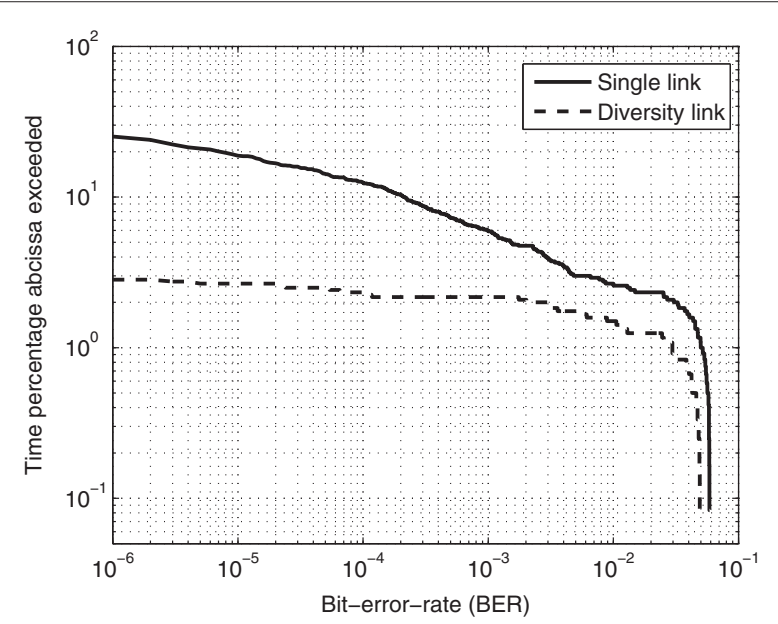

Figure 10 CCDFs of BERs for the combined effect of noise, interferences, and multipath propagation for single and diversity links. The simulation parameters are given in Table 1. impulsive noise, i.e., when $P_{\mathrm{BG}}=0.1, P_{\mathrm{BG}}=0.05$, and $P_{\mathrm{BG}}=0.01$ (in practice, these parameters need to be estimated from measurements) are shown in Figure 11. We can clearly observe from Figure 11 that how the bursty nature of the impulsive noise can affect the performance of industrial WSNs.

\section{Conclusion}

In this article, a complete dynamic wideband channel model for industrial WSN is presented. The model takes into account the noise, interferences, and heavy multipath propagation effects present in harsh industrial environments. A first-order two-state Markov process is adopted to describe the typical bursty nature of the impulsive noise usually present in industrial environments. The interference effects are modeled as multiple narrowband signals operating on the same frequency band as the desired signal. The multipath propagation in industrial environment is described by assuming the scatterers to be uniformly distributed in space within an elliptical region where the transmitting and receiving nodes are located at the foci of the ellipse.

Short-range wireless technologies such as IEEE 802.15.4 in mesh network configuration are widely considered to be cost-effective solution for use in industrial settings. A performance evaluations of the physical layer of IEEE 802.15.4 in terms of BER using the developed dynamic wideband channel model are presented. The results show the combined effect of noise, interferences, and multipath propagation on the BER of industrial WSN. They also demonstrate the advantage of using link diversity (in addition to spread spectrum techniques) to improve the link quality in harsh industrial environments.

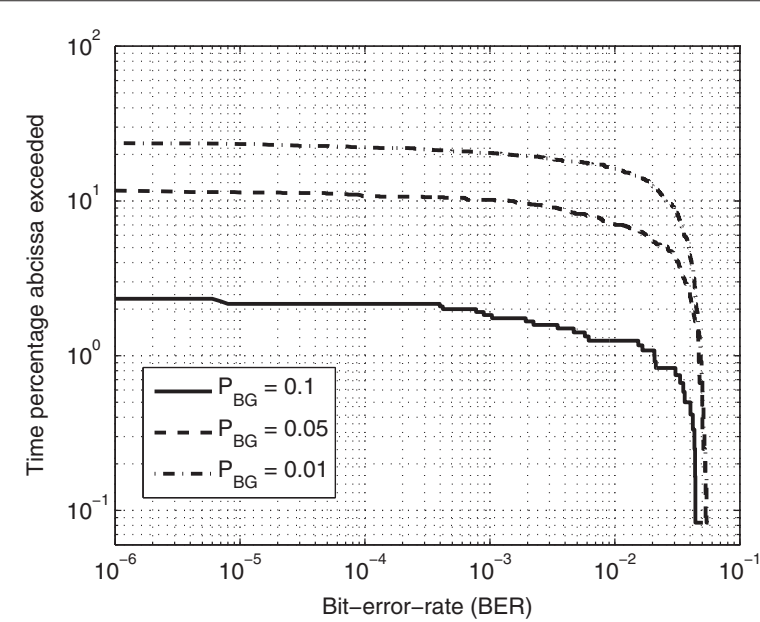

Figure 11 CCDFs of BERs for the combined effect of noise, interferences, and multipath propagation at the output of an MRC when $P_{\mathrm{BG}}=\mathbf{0 . 1}, P_{\mathrm{BG}}=\mathbf{0 . 0 5}$, and $\boldsymbol{P}_{\mathrm{BG}}=\mathbf{0 . 0 1}$. The

simulation parameters are given in Table 1. 
In general, the proposed channel model can be used for generating time series which can be used for successfully designing robust industrial WSNs, and for simulating the performance of WSNs in harsh industrial environments. This enables simulation of for example capacityenhancing techniques such as route diversity, power control, and adaptive coding and modulation.

Future work includes validating the developed dynamic wideband channel model for industrial WSN using RF measurements.

\section{Competing interests}

The author declares that he has no competing interests.

\section{Acknowledgements}

The author would like to thank Gjøvik University College, Norway, for supporting this study.

\section{Received: 1 May 2012 Accepted: 29 August 2012}

Published: 19 September 2012

\section{References}

1. G Simon, M Maroti, A Ledeczi, G Balogh, B Kusy, A Nadas, G Pap, J Sallai, K Frampton, in Proc 2nd Int Conf on Embedded Net sensor Sys. Sensor network-based countersniper system, (Baltimore, MD, November 2004), pp. 3-5

2. J Yick, B Mukherjee, D Ghosal, in Proc 2nd IEEE Int Conf on Broadband Net Analysis of a prediction-based mobility adaptive tracking algorithm, vol 1, (Boston, October 3-7, 2005), pp. 753-760

3. M Castillo-Effen, DH Quintela, R Jordan, W Westhoff, W Moreno, in Proc 5th IEEE Int Caracas Conf on Devices, Circuits, and Sys, Punta Cana, Dominican Republic. Wireless sensor networks for flash-food aletring, (2004)

4. K Lorincz, D Malan, TRF Fulford-Jones, A Nawoj, A Clavel, V Shnayder, G Mainland, M Welsh, S Moulton, in Pervasive Computing for First Response (Special Issue), IEEE Pervasive Computing. Sensor networks for emergency response: challenges and opportunities, (October 2004), pp. 3(4), 16-23

5. T Gao, D Greenspan, M Welsh, RR Juang, A Alm, in Proc 27th IEEE EMBS Annual Int Conf. Vital signs monitoring and patient tracking over a wireless network, (Shanghai, June 17-18, 2005), pp. 102-105

6. D Sexton, M Mahony, M Lapinski, in Proc IEEE Sensors for Industry Conf, Houston, Texas. Radio channel quality in industrial wireless sensor networks, (2005), pp. 88-94, 8-10 February

7. M Alnuaimi, K Shuaib, I Jawhar, in Innovations in Inf Tech, Dubai. Performance evaluation of IEEE 802.15.4 physical layer using MatLab/simulink, pp. 1-5, 19 - 21 November, (2006)

8. KS Low, WNN Win, MJ ER, in Proc Int Conf Comput Modelling, Control Auto, Vienna, Austria 2. Wireless sensor networks for industrial environments, (2005), pp. 271-276, 28-30 November

9. Cisco secure wireless plant: Security and quality of service for industrial environments (2008). http://www.cisco.com/web/strategy/docs

10. IEEE standard, Standard for telecommunications and information exchange between systems - Local area and metropoliatan area networks - Specific requirements Part 15.4: Wireless meadia access control (MAC) and physical layer (PHY) specifications for low rate wireless personal area networks (WPAN) (2003)

11. VC Gungor, GP Hancke, Industrial wireless sensor networks: challenges, design principles, and technical approaches, IEEE Trans. Indus. Elect. 56(10), 4258-4265 (2009)

12. KA Remley, G Koepke, C Holloway, D Camell, C Grosvenor, Measurements in harsh RF propagation environments to support performance evaluation of wireless sensor networks, Sensor Rev. 29(3), 211-222 (2009)

13. TS Rappaport, Characterization of UHF multipath radio channels in factory building, IEEE Trans. Antennas Propagat. 37, 1058-1069 (1989)

14. N Assous, N Lebedev, R Daviot, N Abouchi, in IEEE 14th Int Workshop on Computer Aided Modeling and Design of Communication links and Networks (CAMAD), Pisa, Italy. Wireless sensors for instrumented machines: propagation study for stationary industrial environments, (12 June 2009), pp. 1-5
15. S Luo, N Polu, Z Chen, J Slipp, in IEEE Radio Wireless Symposium. RF channel modeling of a WSN testbed for industrial environment (Phoenix, 16-19, January 2009), pp. 375-378

16. M Sepulcre, JA Palazon, J Orozco, in IEEE Indus Embed Sys. Wireless connectivity for mobile sensing applications in industrial environments (Vasteras, 15-17 June, 2011), pp. 111-114

17. I Howitt, WW Mangesb, PT Kurugantib, G Allgoodc, JA Gutierrezd, JM Conrada, Wireless industrial sensor networks: framework for QoS assessment and QoS management, ISA Trans. 45(3), 347-359 (2006)

18. KL Blackard, TS Rappaport, CW Bostian, Measurments and models of radio frequency impulsive noise for indoor wireless communications, IEEE J. Sel. Commun. 11(7), 991-1001 (1993)

19. D Fertonani, G Colavolpe, in ISPLC. On reliable communication over channels impaired by bursty impulse noise, (2008), pp. 357-362, 2-4 April

20. A Papoulis, Probability, Random Variables and Stocastic Processes. (Mcgraw-Hill College, New York, 1991). ISBN: 978-0070484771

21. Recommendation ITU-R P.1238-6, Propagation data and prediction methods for the planning of indoor radiocommunication systems and radio local area networks in the frequency range $900 \mathrm{MHz}$ to $100 \mathrm{GHz}$, Geneva, 2009)

22. $\mathrm{RH}$ Clarke, A statistical theory of mobile radio reception, Bell Syst. Tech. J. 47, 957-1000 (1968)

23. RJC Bultitude, Measurement characterization and modeling of indoor $800 / 900 \mathrm{MHz}$ radio channels for digital communications, IEEE Commun. Mag. 25(6), 5-12 (1987)

24. SJ Howard, K Pahlavan, Doppler spread measurements of indoor radio channel, Electron Lett. 26(2), 107-109 (1990)

25. JB Andersen, JO Nielsen, GF Pedersen, G Bauch, Doppler spectrum from moving scatterers in a random environment, IEEE Trans. Wirel. Commun. 8(6), 3270-3276 (2009)

26. JC Liberti, TS Rappaport, in Proc IEEE veh Tech Conf. A geometrically based model for line-of-sight multipath radio channels (Spring, 2, 1996), pp. 844-848

27. PM Shankar, Introduction to Wireless Systems. (John Wiley and Sons Inc, New York, 2002). ISBN: 0-471-32167-2

doi:10.1186/1687-1499-2012-297

Cite this article as: Cheffena: Industrial wireless sensor networks: channel modeling and performance evaluation. EURASIP Journal on Wireless Communications and Networking 2012 2012:297.

\section{Submit your manuscript to a SpringerOpen ${ }^{\oplus}$ journal and benefit from:}

Convenient online submission

- Rigorous peer review

- Immediate publication on acceptance

- Open access: articles freely available online

- High visibility within the field

- Retaining the copyright to your article

Submit your next manuscript at springeropen.com 